\title{
Appendizitis? Mit Ultraschall zur Diagnose
}

Kinder haben häufig akute Bauchschmerzen - oft ist es nicht leicht, die Ursache dafür zu finden. Schmerzen im rechten Unterbauch können auf eine Blinddarmentzündung hinweisen, aber auch andere Auslöser sind möglich. Eine sehr genaue Diagnose ermöglicht der Bauchultraschall - das hat jetzt eine weitere Studie gezeigt.

Eine Blinddarmentzündung tritt bei Kindern zwischen sechs und zehn Jahren besonders häufig auf, kann aber auch bei Kleinkindern vorkommen. Bei ihnen ist sie zwar seltener, aber oft besonders schwer zu diagnostizieren. "Zunächst verspüren Erkrankte oft Schmerzen, die vom Bauchnabel ausgehen und sich anschließend in den rechten Unterbauch verschieben", sagt PD Dr. Doris Franke von der Kinderklinik an der Medizinischen Hochschule Hannover. „Häufig kommen Übelkeit und Fieber hinzu. 'Wenn die Beschwerden bei Bewegungen mit Erschütterungen schlimmer werden, beispielsweise beim Hüpfen, dann sollten Eltern mit ihren Kindern umgehend zum Arzt gehen, der mit einer Ultraschall- und Blutuntersuchung abklärt, ob eine Blinddarmentzündung dahintersteckt.

\section{Ultraschall bereits frühzeitig}

Die Sonografie des Bauchraumes ermöglicht bei den kleinen Patienten eine besonders exakte Diagnose - wie eine aktuelle Studie von spanischen Wissenschaftlern zeigt. Marcos PradaArias von der Universitätsklinik Ávaro Cunqueiro in Vigo und seine Kollegen haben für die Untersuchung nach den wertvollsten Instrumenten zur exakten Diagnose einer kindlichen Appendizitis gesucht [1]. Über fünf Jahre hinweg dokumentierten dieWissenschaftler die Daten von 82 Kindern unter fünf Jahren, die mit Verdacht auf Appendizitis in die pädiatrische Notaufnahme der Universitätsklinik in Vigo gebracht wurden. Eine besonders gute diagnostische Klarheit brachte der Bauchultraschall. „Bei Kindern, die mehr als zwölf Stunden unter Beschwerden litten, konnte eine Blinddarmentzündung mittels Ultraschall in dieser Studie zu $100 \%$ diagnostiziert werden“, so Dr. Rüdiger Kardorff, Sektionsleiter Kindergastroenterologie in Duisburg. Auch wenn eine Diagnosesicherheit von $100 \%$ im praktischen Alltag nicht erreicht wird, hat die Sonografie gerade bei den kleinen Patienten eine besonders hohe Aussagekraft viel exakter beispielsweise als die gängige Methode der Blutuntersuchung. Die Expertin empfiehlt, die Ultraschalldiagnostik bereits frühzeitig als Bildgebung zur Abgrenzung verschiedener Differentialdiagnosen anzuwenden.

\section{Rasche Diagnose ist wichtig}

Eine rasche Diagnose ist sehr wichtig, da eine Blinddarmentzündung bei Diagnoseverschleppung lebensbedrohlich werden kann. „Wenn die Appendizitis nicht rechtzeitig erkannt und behandelt wird, kann der entzündete Wurmfortsatz des Blinddarms platzen", sagt der Kinderchirurg Dr. med. Kay Großer, Stellvertretender Leiter der DEGUM-Sektion Pädiatrie. „Dabei können Eiter und Stuhl in die Bauchhöhle gelangen - und so eine lebensbedrohliche Blutvergiftung auslösen."

\section{Sonografie und Sonopalpation}

Die Sonografie des Bauchraumes ist bei Kindern primär empfehlenswert, da sie generell sonografisch besser zu untersuchen sind als Erwachsene und da bei ihnen durch die im Vergleich zu Erwachsenen dünneren Bauchdecken noch mehr Einzelheiten dargestellt werden können. „Organe und Strukturen, von denen das Bauchweh herrühren könnte, sind per Ultraschall besonders gut darstellbar. Unterstützt wird das durch Anwendung der Sonopalpation, bei der mit dosiertem Druck die schmerzhafte Region untersucht wird. Gefahndet wird nach Umgebungsveränderungen, die bei der Entscheidungsfindung zu einer eventuell erforderlichen Operation hilfreich sind", erläutert Großer.

\section{Ultraschall-Kompetenz}

Wesentliche Voraussetzung für eine genaue Diagnose ist neben entsprechender Gerätetechnik die UltraschallKompetenz des behandelnden Arztes: „Nur wenn der Untersucher entsprechend qualifiziert ist, kann er eine Blinddarmentzündung exakt diagnostizieren oder auch hinreichend sicher ausschließen", so Universitätsprofessor Dr. Hans-Joachim Mentzel, DEGUM-Experte und Leitender Arzt der Sektion Kinderradiologie am Universitätsklinikum Jena. Weiterführende invasivere Bildgebung wie die Magnetresonanztomografie, die in dieser Altersgruppe mitunter eine Sedierung erforderlich macht, kann den Kindern so erspart werden.

\section{Literatur \\ 1. (2019) Appendicitis or non-specific abdominal pain in pre-school children: When to request abdominal ultrasound? J Paediatr Child Health. https://onlinelibrary. wiley.com/doi/abs/10.1111/jpc.14617}

\section{Weitere Informationen:}

www.degum.de

Hinweis des Verlags. Der Verlag bleibt in Hinblick auf geografische Zuordnungen und Gebietsbezeichnungen in veröffentlichten Karten und Institutsadressen neutral.

Paediatr. Paedolog. 2020 · 55:102 https://doi.org/10.1007/s00608020-00759-4

(c) Springer-Verlag GmbH Austria, ein Teil von Springer Nature 2020 